\title{
Diagnóstico del origen y la causa de la muerte después de la autopsia médico-legal ( Parte II)
}

\author{
Diagnosis of the origin and cause of death after the medico-legal \\ autopsy (Part II)
}

\section{Resumen}

Una de las tareas más difíciles en patología forense puede ser determinar, después de la autopsia, el origen y la causa de la muerte cuando dichos extremos no están claros o son discutibles. Una autopsia técnicamente perfecta es condición necesaria pero no suficiente para cumplir adecuadamente esta decisión. En el presente trabajo se delimitan claramente los conceptos de causa y mecanismo de muerte, muerte natural y muerte violenta. Se revisan muchos de los aspectos relacionados con el diagnóstico del origen y la causa de la muerte; especialmente el enfoque actual del valor de la autopsia en el diagnóstico, la interacción entre traumatismo y enfermedad, la doctrina de la causalidad, la utilización de la CIE-10, la llamada etiología médico-legal, la autopsia psicológica, la "autopsia blanca" o negativa y el origen de la muerte por complicaciones de la terapéutica, etc., todos ellos se ilustran con ejemplos de casos representativos. Se discute el limitado papel del patólogo forense en España en la determinación de la denominada etiología médico-legal.

Las conclusiones constituyen un conjunto de recomendaciones para el mejor cumplimiento de este trabajo, entre ellas el abandono de las denominadas causas de muerte inaceptables como parada cardiaca, parada cardiorrespiratoria o muerte cerebral, etc., la utilización siempre que sea posible de los diagnósticos de la CIE-10 y del formato del apartado de la causa de la muerte (parte I y II) del modelo internacional de certificado médico de causa de defunción de la OMS. Todo ello como guía breve que facilite un resultado judicial adecuado y aumente la credibilidad del patólogo forense.

Palabras clave: Autopsia médico-legal. Origen de la muerte. Causa de muerte. Mecanismo de muerte. Etiología médico-legal. Muerte natural. Muerte violenta.

\section{Abstract}

One of the most difficult tasks in forensic pathology is deciding the origin and the cause of death after the autopsy when those issues are unclear or debatable. A technically perfect autopsy is a necessary but not a sufficient condition to adequately fulfil this decision. The present paper clearly defines the concepts of cause and mechanism of death, natural and violent death. We review many aspects related to the diagnosis of the origin and cause of death, especially the current approach of the value of autopsy in the diagnosis, the interaction between trauma and disease, the doctrine of causation, the use of ICD-10, the so-called manner of death, the psychological autopsy, the negative autopsy and the origin of death from therapeutic complication, and so on., all of which are illustrated with relevant examples. We also discuss the limited role of the forensic pathologist in Spain in determining the so-called manner of death.

The conclusions include(s) a list of recommendations for the best performance of this work, including the abandonment of the so-called unacceptable causes of death -as cardiac arrest, cardiopulmonary arrest or brain death, and so onand whenever possible the adoption of the classification of ICD-10 and the standard paragraph in which the cause of death is informed (part I and II), as proposed by the WHO international certificate of death. These conclusions intend to be a brief guide to provide a fair judicial outcome and enhance the forensic pathologist's credibility.

Key words: Medico-legal autopsy. Origin of death. Cause of death. Mechanism of death. Manner of death. Natural death. Violent death.

\section{JL. Palomo Rando ${ }^{1}$ \\ V. Ramos Medina ${ }^{2}$ \\ E. de la Cruz $\mathrm{Mera}^{3}$ AM. López Calvo ${ }^{4}$}

${ }^{1}$ Médico Forense. Jefe de Servicio de Patología Forense. ${ }^{2}$ Médico Forense. Jefe de Sección de Histopatología.

${ }^{3}$ Médico Forense. Jefe de Servicio de Laboratorio.

${ }^{4}$ Médico Forense. Servicio de Patología Forense. IML Málaga.

Correspondencia: JL. Palomo Rando Instituto de Medicina Legal Ciudad de la Justicia Fiscal Luis Portero, 6 29010 Málaga

E-mail:

jlpalom045@telefonica.net

Fecha de recepción: 20.SEP.2010

Fecha de aceptación:

12.NOV.2010 


\section{Casos especialmente complejos. Más de una causa de muerte}

Situación especialmente polémica es cuando hay dos o más potenciales causas de muerte. En estos casos pueden ocurrir dos posibilidades:

- Cuando ambas pueden causar la muerte pero puede establecerse una más rápida, más inmediata o sea la que tiene más capacidad letal. Es el caso de una rotura cardiaca post-infarto y una intoxicación por inyección intravenosa de heroína; claramente la rotura cardiaca es más rápidamente letal.

- Cuando no hay medio de determinar cuál de ellas realmente pudo causar la muerte, pues ambas tienen similar capacidad letal y, por consiguiente, pueden ser igualmente responsables de la muerte. Es el caso de signos objetivos pero incompletos de estrangulación a lazo e intoxicación aguda por cocaína. En este caso, algunos ${ }^{1}$ opinan que optar por una sola causa puede conducir a una posición insostenible debido a la extrema simplificación; especialmente si, como en este caso, en una de ellas hay una responsabilidad criminal. Relacionarlas como dudosas no les parece muy adecuado. Entonces recomiendan establecer la causa de la muerte de forma descriptiva (muerte violenta con signos de presión aplicada en el cuello e intoxicación aguda por cocaína) que abarque a ambas causas. A nosotros nos parece más adecuado decir que cualquiera de ellas pudo causar la muerte, pero que no puede establecerse con seguridad cuál de ellas la causó.

En el caso ya expuesto de signos de cardiopatía isquémica y niveles supraterapéuticos de opiáceos y benzodiacepinas, sin otra información, habría dos soluciones aceptables: una, establecer un origen violento por intoxicación y la cardiopatía como factor contribuyente; la otra, establecer un origen indeterminado, relacionando ambas causas. En los ejemplos citados, cuando coexisten una causa natural y otra violenta, nos parece más adecuada la primera opción, es decir, establecerla como muerte violenta, pues la causa natural ha sido tolerada durante mucho tiempo.

\section{Miscelánea}

Existen una serie de muertes, muy poco frecuentes en nuestro medio, que plantean dudas sobre si establecer su origen como natural o violento.
Las enfermedades infecciosas transmitidas por vectores (paludismo, fiebres recurrentes y exantemáticas transmitidas por piojos y garrapatas, rabia, etc.) son consideradas por algunos como naturales ${ }^{36}$. Nuestro criterio es que las enfermedades infecciosas que afectan a grandes grupos de población y están asociadas a transmisión por picaduras de artrópodos son naturales. No así la rabia (CIE-10, A82), cuya transmisión habitual es la mordedura de mamíferos que se considera una violencia.

Las producidas por el efecto tóxico de las mordeduras de serpientes, arácnidos o peces venenosos plantean menos dudas y casi todas las publicaciones las consideran violentas, incluidas las producidas por anafilaxia tras picadura de abejas y avispas.

La muerte por anafilaxia plantea dudas. La anafilaxia a alimentos (casos de anafilaxia mortal por alergia a frutos secos, especialmente cacahuetes) o medicamentos son valoradas por algunos ${ }^{36}$ como violentas (accidentes). Nuestra opinión es que la anafilaxia por alimentos es natural. La alergia es una enfermedad e ingerir alimentos normales es un proceso natural, por consiguiente, no se evidencia ninguna violencia. La anafilaxia por medicamentos es una complicación de la terapéutica.

Infecciones adquiridas como consecuencia de un accidente de trabajo (infecciones en personal de laboratorios de bacteriología o personal sanitario en general, HIV por punción con aguja, bien documentada) serían violentas (accidentales).

\section{El problema de las etiologías médico-legales}

Los patólogos forenses de los países en los que están obligados a establecer la etiología médico-legal después de la autopsia manifiestan por ello notables discrepancias e inconvenientes ${ }^{35,37}$.

En 1997 se publicó una encuesta enviada a los más de 700 patólogos forenses miembros de la NAME (Nacional Association of Medical Examiners) a la que sólo contestaron completamente 198 y en la que se les pedía que clasificaran la etiología médico-legal (manner) de 23 casos típicos, aunque polémicos, de patología forense, junto a algunas otras cuestiones ${ }^{35}$. El resultado puso de manifiesto las discrepancias a la hora de determinar el tipo, pues solamente en 11 de los 23 casos estuvieron de acuerdo más del $80 \%$ de los que respondieron a la encuesta; el $100 \%$ de acuerdo entre los encuestados sólo se produjo en la valoración de un caso, y en dos casos la concordancia de las calificaciones no alcanzó ni el 50\%. 
En un panel organizado para analizar los resultados se discutió sobre si la muerte de un sujeto jugando a la ruleta rusa debía considerarse un accidente o un suicidio, apuntando algunos que dicha consideración estaría en función del número de balas en el tambor del revólver ${ }^{38}$.

Algunos sugirieron en sus respuestas que la clasificación de la etiología debía ser eliminada del certificado estándar de defunción estadounidense.

Este estudio también puso de manifiesto que los familiares de los fallecidos por suicidio reclaman con cierta frecuencia contra las etiologías médico-legales, bien por el estigma que se vincula a las muertes suicidas o por razones económicas, pues muchas pólizas de seguros de vida tienen cláusulas de exclusión por muerte suicida. Además, también hay casos de muertes naturales que son motivo de litigio, pues en ellas se excluyen muchas indemnizaciones que sólo se hacen efectivas si la muerte fue accidental. Esto último también viene ocurriendo en España.

El $21 \%$ de los patólogos reconoció que había sido amenazado de demandas por la etiología médicolegal establecida y un $9 \%$ que habían sido efectivamente demandados en una o más ocasiones, generalmente en relación con la clasificación de suicidio de determinadas muertes, siendo generalmente los familiares, y en menos ocasiones la policía, fiscales o compañías de seguros, los que habían intervenido en estas presiones.

\section{La "autopsia blanca" o negativa}

En los grandes centros de patología forense se alcanza un 5\% de autopsias blancas, excluido el síndrome de la muerte súbita del lactante. En contra de lo que cabía esperar, este resultado es más frecuente entre experimentados patólogos que entre los jóvenes, que piensan que no encontrar causa de muerte pondría en tela de juicio su preparación ${ }^{20}$.

Si al final de todos los estudios no se encuentra una causa de muerte ${ }^{39,40}$, la conclusión debe ser que en el estado actual del conocimiento médico y científico no se ha podido llegar a un diagnóstico que explique la muerte. Si se han excluido lesiones traumáticas e intoxicaciones se puede añadir que es más probable que se trate de una muerte natural que de una violencia externa.

Es fundamental la honradez del patólogo en estas situaciones, se deben reconocer las limitaciones y no recurrir a causas inaceptables, como parada cardiorrespiratoria, fallo cardiaco, o a eventos agónicos, como la broncoaspiración de contenido gástrico, o procesos indemostrables como parada cardiaca refleja, inhibición vagal o sofocación.

Un parámetro más del rigor científico en el trabajo del patólogo forense sería conocer el porcentaje de "autopsias blancas" en su haber.

\section{Autopsia psicológica}

Las indicaciones y utilidad de la autopsia psicológica deben ser bien conocidas por el patólogo forense, pues es un instrumento que, en manos expertas, puede ayudar en los casos de muertes sospechosas en los que las circunstancias que rodearon o condujeron a la muerte son confusas, es decir, hay dudas sobre si la muerte fue accidental, suicida u homicida ${ }^{41}$.

Hay muchos casos médico-legales en los que la personalidad, motivación y actuación de la persona fallecida deben ser estudiadas. Estos aspectos son especialmente importantes cuando hay primas de seguros de vida que son anuladas en casos de suicidios y, desde luego, en los crímenes en los que se puede cuestionar si el fallecido pudo contribuir a su propia muerte en alguna forma.

La autopsia psicológica fue desarrollada en la década de 1950 por RE. Litman, ES. Shneidman y NL. Farberow, psiquiatra y psicólogos respectivamente, pertenecientes al Centro de Prevención del Suicidio de Los Ángeles (California) ${ }^{42}$. En el sótano del hospital en el que trabajaban encontraron una caja con más de 200 notas de suicidas. Fue el estudio de estas notas y otros aspectos de dichos suicidios lo que dio lugar a varias publicaciones y a un prestigio reconocido, tras el que fueron requeridos por compañías de seguros para que les ayudasen a determinar si los titulares de una póliza habían muerto accidentalmente o se había tratado de un suicidio.

Shneidman (1994) define la autopsia psicológica como la reconstrucción retrospectiva de la vida de un individuo centrada en su muerte, es decir, los rasgos de su vida que informan sobre sus intenciones en relación con su propia muerte, los indicios de qué tipo de muerte fue, el grado (si lo hubo) de su participación en su propia muerte y por qué la muerte ocurrió en ese momento.

La técnica consiste esencialmente en entrevistar a familiares, amigos, compañeros de trabajo, médicos, empleados, etc., incluso maestros y camareros que pudieran facilitar información relevante para reconstruir los antecedentes del fallecido, las relaciones 
personales y el estilo de vida. Especial atención se presta a los eventos inmediatos que precedieron a la muerte. Toda esta información es revisada, junto con el médico forense, por el equipo que, bajo la dirección del Juez instructor, investiga la muerte para establecer la etiología médico-legal. Shneidman desarrolló un protocolo de 16 puntos que no es muy diferente al que utilizaría cualquier médico al hacer una detallada historia clínica, la única diferencia es que en este caso se centra en la reacción ante el estrés, relaciones interpersonales, pensamientos revelados, experiencias y actuaciones inmediatamente anteriores a la muerte. Entre dichos puntos cabe destacar la causa, método y otros detalles pertinentes de la muerte: antecedentes personales y familiares (hermanos, matrimonio, enfermedades, tratamientos, intentos de suicidio, etc.), papel del alcohol y las drogas en la vida y muerte de la víctima, modo de reacción al estrés emocional y periodos de desequilibrio, cambios en los hábitos de vida anteriores a la muerte (comida, hobby, etc.). Mucho más exhaustivo es el protocolo de Ebert (1987) que analiza 26 ítems, cada uno a su vez con varios puntos.

Hay pocas investigaciones sobre la confianza y validez de la autopsia psicológica, por lo que se recomienda a los profesionales que la manejan mucha precaución en el empleo de evaluaciones de reconstrucción psicológica. A pesar de sus muchos puntos débiles en los procedimientos utilizados y la falta de investigaciones consistentes que apoyen su validez, es evidente que se han presentado ante muchos tribunales en Estados Unidos donde frecuentemente se han tenido en cuenta.

\section{Dificultades en la elaboración de la opinión y grado de certeza}

La toma de decisiones para formular la causa de la muerte debe ser un proceso riguroso que compare todas las perspectivas cuando se enfrenta a un caso difícil.

Una causa frecuente de error es cuando el patólogo se aferra a un hallazgo particularmente interesante y desecha otros indicios igualmente convincentes, que apuntan a un diagnóstico contrario. Esta conducta era lo que Moritz ${ }^{43}$ Ilamaba sustituir una interpretación científicamente defendible por la intuición y que puede ser motivo de un juicio penal o civil injustificado.

Otra causa de error es el fracaso en apreciar los diferentes grados de probabilidad dando lugar a que una idea especulativa se presente como una probabilidad razonable. Las especulaciones son posibilidades que no se basan en la razón, son sutilezas o hipótesis sin base real.

En ocasiones el grado de certidumbre puede ser bajo $y$, salvo en casos de alteraciones incompatibles con la vida, de lo que se trata es de establecer una causa (la mejor) que explique razonablemente la muerte a la luz de los conocimientos actuales de la patología basada en la evidencia. Esto es huir de la especulación, no basarse exclusivamente en la propia experiencia y hacerlo sobre todo en trabajos de revisión de las publicaciones con mayor índice de impacto y textos más aceptados por la comunidad científica ${ }^{44}$.

Algunos patólogos forenses norteamericanos ${ }^{16,24}$ tratan de sistematizar los grados de certeza de acuerdo con las normas vigentes en su país sobre el grado de convicción judicial necesaria para establecer la culpabilidad en un juicio penal o aceptar la demanda en un procedimiento civil. En los casos en que haya responsabilidad criminal debe haber un grado de certeza médica razonable, es decir, que razonablemente puedan excluirse otras posibilidades diagnósticas 0 , dicho de otra forma, otra contingencia imaginable es imposible. Aunque el grado de certeza razonable no tiene una probabilidad numérica exacta se admite que es igual o superior al 90\%. Ello deriva del precepto legal en EE.UU. de que la culpabilidad penal ante el jurado debe quedar probada más allá de toda duda razonable. Por supuesto, no se permite la especulación.

En los casos que exista sólo responsabilidad civil de terceros o únicamente se trate de hacer un diagnóstico correcto, la norma sería que dicho diagnóstico sea más probable que improbable, es decir, que las otras posibilidades diagnósticas sean menos probables que nuestro diagnóstico; un grado de certeza de al menos el $51 \%$.

Un grado algo menor del 50\% de probabilidad sería una posibilidad razonable y son las que pueden ser discutidas en juicios orales. También son aceptadas para estos casos de menor seguridad las expresiones "compatible con" y "posible y muy probable"24.

Patólogos especializados en muerte súbita cardiaca establecen el grado de certeza diagnóstica en una escala de tres: diagnóstico cierto, altamente probable e incierto ${ }^{45}$. En otras publicaciones fijan la seguridad diagnóstica en cuatro grados: probabilidad elevada, media, baja o muy baja ${ }^{46}$. 


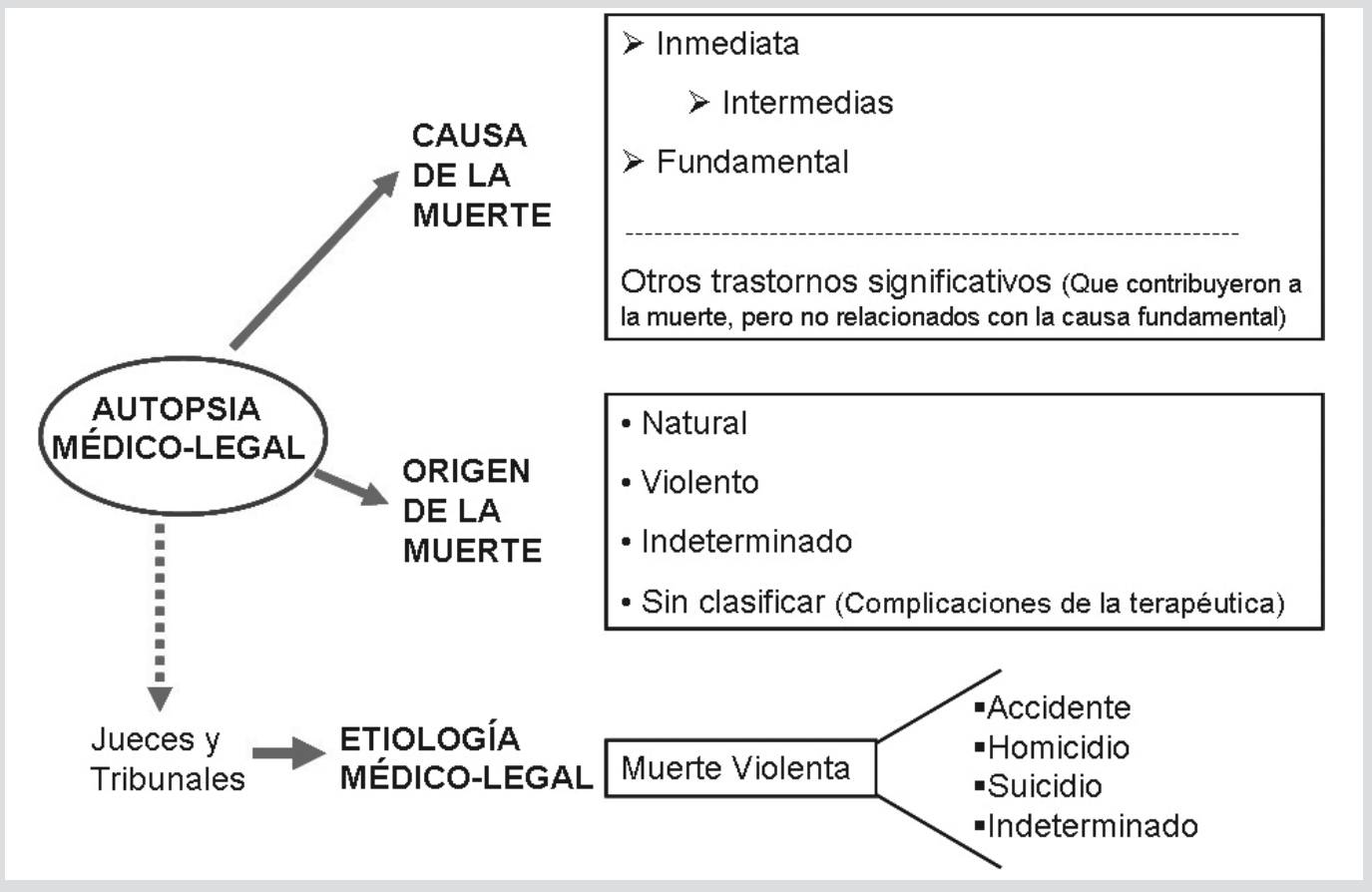

Figura 3.

\section{Conclusiones}

Las conclusiones del presente trabajo pueden considerarse como recomendaciones para establecer adecuadamente el origen y la causa de la muerte después de la autopsia médico-legal (Figura 3):

- Se debe establecer siempre el origen natural o violento de la muerte y, si no se tiene un grado razonable de certeza, declarar que dicho origen es indeterminado.

Para las muertes que se presentan directamente por la aplicación de procedimientos diagnósticos o terapéuticos, es mejor un origen sin clasificar por complicación de la terapéutica.

- La causa de la muerte después de la autopsia debería, siempre que fuera posible, expresarse utilizando la nomenclatura de la Clasificación Internacional de Enfermedades de la OMS (CIE10), de forma que pudiera ser codificada para las estadísticas vitales.

- Es recomendable, siempre que sea posible, adoptar en la conclusiones de los informes de autopsia el formato del apartado de la causa de la muerte (parte I y II) del modelo internacional de certificado médico de causa de defunción; indicando el intervalo aproximado entre el inicio del trastorno y la muerte.

- Lo más importante es consignar la causa fundamental, básica o inicial. No aferrarse constantemente al rígido esquema causa inmediata/causa fundamental, que puede llevar a decir causas o mecanismos erróneos. No siempre hay una causa inmediata y otra fundamental. En ocasiones no hay una cadena de causas, o hay una inmediata y varias intermedias que deben expresarse por orden inverso al cronológico en la producción de la muerte.

- Cuando no se utilicen los diagnósticos de la CIE10, debe emplearse la nosología más utilizada en la bibliografía médica actual.

- Si en el momento de finalizar la autopsia no se tiene un mínimo de seguridad en el origen y la causa, dejar los mismos pendientes de conocer el resultado de los estudios complementarios solicitados. No olvidar que existe la "autopsia blanca" o negativa.

- No utilizar las denominadas causas de muerte inaceptables, inespecíficas y carentes de significado, o sea que nada dicen sobre la causa 
de la misma, como parada cardiaca o cardiorrespiratoria, muerte cerebral, etc. Eventos que no son causa ni siquiera mecanismos de muerte y, por supuesto, nada dicen de lo uno ni de lo otro.

- Tampoco es aceptable utilizar como causas fundamentales de muerte las que expresan sólo mecanismos fisiopatológicos, como insuficiencia renal, edema pulmonar, insuficiencia hepática, fracaso multiorgánico, etc. Sí podría establecerse un mecanismo como causa inmediata o intermedia de muerte (no como causa fundamental o inicial) cuando, de no hacerlo, el caso quedara confuso, pero, insistimos, los mecanismos no son causas de muerte.

- Siempre que sea posible emplear diagnósticos morfológicos o estructurales: hemopericardio, mejor que taponamiento cardiaco; traumatismo craneoencefálico abierto, laceración cerebral, mejor que destrucción de centros vitales. El diagnóstico de fibrilación ventricular sólo puede hacerse con rigor si el sujeto estaba conectado a un monitor.

- No es correcto utilizar causas de muerte que pueden tener indistintamente un origen natural o violento, por ejemplo, hemorragia intracraneal, pues así expresado puede referirse a una hemorragia cerebral hipertensiva de origen natural o a un hematoma subdural agudo por un traumatismo craneoencefálico.

- No se debería incluir en las conclusiones de los informes de autopsia la llamada etiología médico-legal. En España esta determinación le corresponde a la autoridad judicial.

\section{Bibliografía}

36. Hanzlick RL. Medical certification of death and cause-of-death statements. En: Froede RC (ed.). Handbook of Forensic Pathology. $2^{\mathrm{a}}$ ed. Northfield, Illinois: College of American Pathologists 2003.

37. Hanzlick R. Lawsuits against medical examiners or coroners arising from death certificates. Am J Forensic Med Pathol. 1997;18(2):119-23.

38. Goodin J, Hanzlick R. Mind your manners. Part III: Individual scenario results and discussion of the $\mathrm{Na}$ tional Association of Medical Examiners Manner of Death Questionnaire, 1995. Am J Forensic Med Pathol. 1997;18(3):228-45.

39. Molina Aguilar P, Dasí Martínez C, Gisbert Grifo MS. La «autopsia blanca». Revista Española de Patología. 2004;37(1).

40. Adelson L. No anatomic cause of death; the enigma of the forensic pathologist. Conn State Med J. 1954;18(9):732-8.

41. García Pérez T. La autopsia psicológica en las muertes violentas. Rev Esp Med Leg. 1999;XXIII(8687):75-82
42. Canter D, Cremer M. The psychological autopsy. En: The Pathology of Trauma. 3rd ed. Londres: Edward Arnold 2000.

43. Moritz AR. Classical mistakes in forensic pathology: Alan R. Moritz (American Journal of Clinical Pathology, 1956). Am J Forensic Med Pathol. 1981; 2(4):299-308.

44. Pollanen M. Forensic Pathology in Ontario-the Current Status. 2008 Annual Education Course for Coroners and Pathologists. Oct 30- Nov 1, 2008. Toronto. ON. Canada.

45. Basso C, Burke M, Fornes P, Gallagher PJ, de Gouveia RH, Sheppard M, Thiene G, van der Wal A; Association for European Cardiovascular Pathology. Guidelines for autopsy investigation of sudden cardiac death. Virchows Arch. 2008;452(1):11-8. Epub 2007 Oct 20. En lengua española en: Basso C, et al. Guías para la práctica de la autopsia en casos de muerte súbita cardíaca. Cuad Med Forense 2009;15(55):7-16. Disponible en: http://scielo. isciii.es/ scielo. php?script= sci_arttext\&pid=S1135-76062009000100004 \&lng $=$ es\&nrm =iso (acceso 02-02-2010) doi: $10.4321 / \mathrm{S} 1135-76062009000100004$.

46. Sheppard M, Davies MJ. Practical Cardiovascular Pathology. Londres: Arnold 1998. 